\title{
Analyzing effects of transport policies on travelers' rational behaviour for modal shift in Denmark
}

\author{
Ahanchian, Mohammad; Gregg, Jay Sterling; Tattini, Jacopo; Karlsson, Kenneth Bernard
}

Published in:

Case Studies on Transport Policy

Link to article, DOI:

10.1016/j.cstp.2019.07.010

Publication date:

2019

Document Version

Peer reviewed version

Link back to DTU Orbit

Citation (APA):

Ahanchian, M., Gregg, J. S., Tattini, J., \& Karlsson, K. B. (2019). Analyzing effects of transport policies on travelers' rational behaviour for modal shift in Denmark. Case Studies on Transport Policy, 7(4), 849-861. https://doi.org/10.1016/j.cstp.2019.07.010

\section{General rights}

Copyright and moral rights for the publications made accessible in the public portal are retained by the authors and/or other copyright owners and it is a condition of accessing publications that users recognise and abide by the legal requirements associated with these rights.

- Users may download and print one copy of any publication from the public portal for the purpose of private study or research.

- You may not further distribute the material or use it for any profit-making activity or commercial gain

- You may freely distribute the URL identifying the publication in the public portal 


\title{
Analyzing effects of transport policies on travelers' rational behaviour for modal shift in Denmark
}

\author{
Mohammad Ahanchian*, Jay Sterling Gregg, Jacopo Tattini, Kenneth Bernard Karlsson \\ Department of Management Engineering, Technical University of Denmark, Produktionstorvet, Building 426, 2800 Kongens Lyngby, Denmark
}

\section{A R T I C L E I N F O}

\section{Keywords:}

Modal shift

Tangible cost

Value of travel time

\begin{abstract}
A B S T R A C T
In this paper, an innovative agent-based model is developed to simulate emergent patterns arising from individual actions to analyze opportunities for modal shift in Denmark (ABMoS-DK). The modeling process simulates the interaction between travelers (agents) and the network, and applies a heuristic algorithm to model travelers' rational decision making process based on tangible costs (ticket price, fixed and variable operation and maintenance costs) and Value of Travel Time (VOTT) explained by Value of Time (VoT), travel time and level of service. The traveler is described by a set of socio-economic attributes (income, family structure, place of residence, car/bike ownership) and the utility is derived from properties of alternative modes to determine whether to use non-motorized, public or private transport. Fluctuation of tangible costs and value of travel time can provide comparative advantages to the alternative modes and has the potential to affect the utility of the mode derived by traveler and change mode choice decision. A set of "pull" and "push" policy scenarios are formulated to help us understand how different factors affect mode choice in transport. We find that disincentivizing private cars has the highest potential for shifting from car use followed by incentives for sustainable modes and expansion of public transport infrastructure. The paper concludes that capturing rational behavioural features of consumers with fine level of heterogeneity in modelling will help to better understand the dynamics of the transportation system and consequently assist policy makers to better identify and target consumer groups with the highest shift potential.
\end{abstract}

\section{Introduction}

The Danish government has adopted the ambitious goal of becoming independent of fossil fuels by 2050 (The official website of Denmark, 2017). While renewable energy is increasingly deployed to meet power and heat demands in Denmark, the transport sector still depends highly on petroleum products and is regarded as the most complicated sector to decarbonize. In 2010, the transportation sector accounted for approximately $23 \%$ of energy-related $\mathrm{CO}_{2}$ emissions worldwide (Sims et al., 2014) and about 36\% in Denmark (IEA, 2016), $60 \%$ of which are from passenger vehicles (Winther, 2015). The significant challenges faced in moving towards a long-term decarbonization of the transportation sector include the increase of transport demand, lack of available alternatives to fossil fuels, limits to vehicle efficiency, fuel standards and heterogeneity of consumers' behaviour.

The Nordic Energy Technology Perspectives report (IEA, 2016) recommended modal shift as one of the key mechanisms for decarbonizing the transport sector in Nordic countries by 2050. Modal shift takes place when one mode has a comparative advantage over another mode (for instance, in the level of service) and promotes a behavioural change in the travelers' decision. Therefore, modal shift is fundamentally a behavioural change: e.g., shifting to non-motorized transport, increasing the occupancy factor of private vehicles, and higher utilization of public transportation. The focus in modal shift is aimed towards travelers, since freight transportation is more constrained, depending on the market trends and policies that are in effect (Baindur and Viegas, 2011).

Road transportation, is a complex socio-technical system consisting of different components and subsystems in social and technological areas (Adelt et al., 2018). The transport system is often conceptualized as having three components: vehicles or equipment that move objects (people, goods); guideways or what the vehicles move along; and an operation plan or a set of procedures by which objects and vehicles are moved over guideways (timetables, control systems, etc.) (Boyce, 2005). As such, the factors affecting modal choice are the existing infrastructure, socio-demographic factors and the use of policy tools (Hammadou and Papaix, 2015). However, Barisa (2016) argued that this conceptualization excludes users and the complexity of their

\footnotetext{
* Corresponding author.

E-mail address: ahanchian@gmail.com (M. Ahanchian).
} 
heterogeneous decision-making from the system. Capacity and frequency of service of public transportation constrain urban traffic (Hager et al. 2015) and policy makers would want to ensure that the existing infrastructure is sufficient to accommodate (Chen and Liu, 2018) travelers.

The Energy-Environment-Economy-Engineering (E4) models are tools developed for long-term energy planning and determining leastcost decarbonization pathways (Chiodi et al., 2013; Føyn et al., 2011; McCollum et al., 2012; Yang et al., 2015) by representing technology changes while suffering limited ability to fully evaluate the influence of behavioural changes on the energy system. E4 models that are currently used to assess and support energy and climate policies at national and EU level, do not fully integrate and represent the new challenges posed by the energy transition such as behavioural aspects. So, there is a need to enhance their capabilities to analyze both policy implementation aspects and the design of long-term low-carbon transition. Due to the lack of representation of consumers' behaviour in E4 models, the contribution of modal shift to GHG emissions reduction was initially evaluated through "what-if" analyses, which assessed the effect of exogenously assumed levels of transfer of mobility demand from one mode to another on the environment (GEA writing team, 2012; IEA, 2009). In a review of E4 models, Schäfer (2012) concluded that accounting for behaviour changes in E4 models is "indispensable" when developing overarching climate change mitigation strategies for the transport sector.

Several researchers have attempted to integrate transport behavioural features in bottom-up (BU) optimization E4 models. For this class of models, Venturini et al. (2018) recognized two main approaches to incorporate behaviourally realistic modal shift. One consists in linking the BU E4 model with an external transport model that handles the behavioural features and determines modal shares (E3MLab, 2014; Waisman et al., 2013; Girod et al., 2012; Brand et al., 2012). The other approach endogenously assesses modal shift within an energy system model, by enlarging the traditional model structure to include transport-specific variables and transport infrastructure (Daly et al., 2014; Pye and Daly, 2015; Tattini et al., 2018a; Tattini et al., 2018b). These studies attempted to identify the limits for the travel time that users are willing to spend for commuting, as well as the budget they are willing to commit to meet transport demand: TravelTime Budget (TTB) and Travel-Money Budget (TMB) respectively (Schäfer and Victor, 2000). Typically, people are willing to spend an average of $1.1 \mathrm{~h} /$ day on commuting and devote only a small fraction of the households' total budget (approximately $3-5 \%$, for households that do not own a personal car) towards transportation (Schäfer and Victor, 2000). When income increases, users shift to faster modes of transportation; wealthier societies have increased mobility levels (Schäfer and Victor, 2000).

Discrete choice model is a methodological approach to simulate how users select the desired mode of transport for meeting travel demand. Several studies in the field of travel mode choices have used discrete choice models (Chikaraishi and Nakayama, 2016), multinomial logit regression (Arbués et al., 2016; Thrane, 2015), nested logit (Lu et al., 2015), generalized extra value, mixed logit and probit (Can, 2013; Eboli et al., 2016) based on the random utility maximization theory (McFadden, 1978). Such analysis models the choice of a decision maker among a set of finite alternatives. These approaches have some limitations, such as: i) the strict model structure needs to be specified in advance; ii) they are unable to model non-linear systems; and iii) they consider only conditions that hold across an entire population of observations (Shukla et al., 2013 In: Maggi and Vallino, 2016). However, due to heterogeneity of consumers and complex decision-making process based on a large number of parameters, behavioural changes concerning mode of transport are subject to high degree of uncertainty. Therefore, discrete choice models are not sufficient to model the complex behaviour involved in modal choice decisions. Moreover, researchers are usually interested to investigate the impacts of transportation plans on the behaviour of individual households, persons or subgroups (Shirzadi-Babakan et al., 2015).

An alternative approach is agent-based modeling (ABM), which is capable of simulating a large number of heterogeneous individuals with different attributes, characteristics, behaviour and perception represented as agents. Agent-based modeling and simulation (ABMS) is an approach for modeling complex systems composed of collection of autonomous decision-making entities called agents that execute various behaviours appropriate for the system they represent (Bonabeau, 2002; Macal and North, 2010). Ahanchian and Biona (2017) provided an extensive list of researches using $\mathrm{ABM}$ approach within different contexts.

During the past decade, several studies have used agent-based modeling approach within the context of traffic. Adelt et al. (2018) proposed a general-purpose simulation framework, "Simulation of the governance of complex systems (SimCo)" to model and analyse interaction processes in networked systems and study the complexity of socio-technical systems where a large number of actors act and interact, producing emergent effects at the system level and are only partly controllable. The study demonstrated that ABM is a useful method to tackle the issue of governability of complex socio-technical systems and thus to stimulate the inconclusive debate which has been going on in sociology and political science for decades. The framework has been applied to the case of road transportation. Many researchers used the Multi-Agent Transport Simulation (MATSim) to simulate the dynamics of transport system such as route choice (Maciejewski and Nagel, 2012) and bicycle traffic (Ziemke et al., 2018). Kaddoura and Nagel (2016) analyzed the heterogeneous values of travel time saving, Ben-Dor et al. (2018) assessed the impacts of dedicated bus lanes on urban traffic congestion and modal split and Hörl et al. (2018) showed that implementing a discrete mode choice model may drastically increase the convergence speed of the simulation. Moreover, AnyLogic tool is used to develop agent-based model to simulate shared-taxi system (Martinez et al., 2014), carsharing (Martinez et al., 2016), shared self-driving urban mobility (Martinez and Viegas, 2017) and automated vehicles as last mile connection of train trips (Scheltes and Correia, 2017) and analyzed the impacts of these developments on transport system.

Table 1 lists studies that use ABM as a tool for analysing mobility behaviour. The review of the literature shows the importance of incorporating behavioural aspects in traffic related analysis and the capability of agent-based modeling approach to simulate behavioural aspects. However, the studies regarding mode choice consider only an urban area (city) as a geographical scope. Maggi and Vallino (2016) in their critical review of literature on ABM focusing on transport concluded that there is still a gap in urban transport agent-based modelling: They are usually focused on sub-categories of city inhabitants, such as school pupils, students, pedestrians or car owners, without a systemic view. Moreover, they discussed the need to implement real surveys in order to calibrate the ABMs using first-hand data (Maggi and Vallino, 2016).

In our study, we developed a novel agent-based model, Agent-Based Modal Shift Simulation for Denmark (ABMoS-DK), and apply it to simulate modal shift in the inland transportation sector with a systematic approach. Within ABMoS-DK, agents are independent in making decision while there is a feedback from agents' action and consequently indirect interaction between agents for choosing the mode emerges such as "SimCo" framework developed by Adelt et al. (2018) and ABM package of TRansportation ANalysis SIMulation System (TRANSIMS) (Bonabeau, 2002). However, as far as the authors are aware, there is no previously published work presenting agent-based modeling approach with the focus on utility maximization based on defining the direction and importance of the effect of tangible costs and Value of Travel Time (VOTT). Moreover, the novelty of ABMoS-DK is capturing the realistic heterogeneity of travelers' rational decision-making and integrating several dimensions of mobility parameters such as travel cost, travel time, emerging congestion, level of service of modes, availability of 
Table 1

Literature containing $\mathrm{ABM}$ as a tool for transport studies.

\begin{tabular}{|c|c|c|c|}
\hline Reference & Geographic Scope & Focus & Input data \\
\hline Dia, 2002 & Brisbane, Australia & Modelling individual driver behaviour & Behavioural survey of drivers \\
\hline Shafiei et al. 2012 & Iceland & Predicting the evolution of market share of electric vehicles & CreditInfo report \\
\hline Mallig et al. 2013 & Stuttgart, Germany & Modeling travel demand in Stuttgart region & Travel survey and official statistics \\
\hline Fagnant and Kockelman, 2014 & $\begin{array}{l}\text { A hypothetical mid-size } \\
\text { city in US }\end{array}$ & Shared autonomous vehicles and environmental implications & US National Household Travel Survey \\
\hline Novosel et al. 2015 & Croatia & Simulate hourly distribution of transport demand & Official data of the region \\
\hline Hager et al. 2015 & Stuttgart, Germany & Modeling the traffic behavior in growing metropolitan areas & Household survey and statistics \\
\hline $\begin{array}{l}\text { Shirzadi-Babakan and Taleai, } \\
\qquad 2015\end{array}$ & Tehran, Iran & $\begin{array}{l}\text { Evaluate impacts of different transport development plans on choices } \\
\text { of residential location and commuting mode of tenant households }\end{array}$ & Survey and official statistics \\
\hline Zou et al. 2016 & Beijing & Predict mode choice and departure time changes & Behaviour survey of travelers \\
\hline Djavadian and Chow, 2017 & Oakville, Ontario & $\begin{array}{l}\text { Modeling 'Mobility as a Service' with a two-sided flexible transport } \\
\text { market }\end{array}$ & $\begin{array}{l}\text { Network data for Oakville and } \\
\text { Transportation Tomorrow Survey }\end{array}$ \\
\hline
\end{tabular}

mode and capacity saturation within the context of modal choice. ABMoS-DK is used to address the following research questions:

1. How effective are strategies for influencing the travelers' decision on choosing the mode of transport?

2. How much is the maximum shift potential from the viewpoint of travelers without considering technological changes?

3. Which groups of agents (e.g., geographical zones, travel demand length, urbanization pattern and income groups) are most sensitive to various strategies for incentivizing modal shift?

\section{Data and methods}

\subsection{Data}

Based on the Great Belt Corridor, Denmark is divided to East (DKE) and West (DKW) regions. According to settlement patterns, each region is further divided into urban (U), suburban (S) and rural (R) areas (Eurostat, n.d.). Fig. 1 shows the structure for the data collection and how it relates to the calculations done in ABMoS-DK. The Danish National Travel Survey (also denominated TU survey), an interview-based survey that documented the travel behaviour of the Danish population by recollecting mobility diaries, and socio-economic data from 2006 to present (Christiansen and Skougaard, 2015) is used to capture the attributes of travelers. The TU Survey interviewed 23,753 targeted travelers in 2010 and collected data on their personal income, household structure, trip details including timing, origin and destination. This resulted in a database with 29,089 distinct journey records that included the distance, the mode, timing and socio economic data on the person making the trip (TU Survey, 2018). The socio-economic characteristics on the household level (i.e., annual income, car/bike ownership and place of residence), individual attributes (i.e., age, gender, education level and etc.) and the trip characteristics (i.e., trip length, departure time and trip purpose) were assigned to agents through an SQL database.

The Danish Land Transport Model (LTM) was used (http://www. landstrafikmodellen.dk) (Rich and Hansen, 2015) as a supporting model of this study to determine the properties of different modes. LTM is a four-stage simulation transport model of Denmark (Rich, 2015), which represents all transport activities within, into, and through Denmark (Jensen et al., 2017). LTM was used to quantify Value of Time (VoT), average speed of each mode and average congestion time across urbanization areas, penalty parameters of congestion, in-vehicle, access/egress and waiting time together with the annual inland transport demand disaggregated on urbanization type in each region. Shares of transport modes based on passenger-kilometer in 2015 are presented in Fig. 2. The private cars are responsible for the majority of travel demand $(83.7 \%)$, all public transits take $12.3 \%$ and the train has the highest share (7.0\%) followed by bus (3.0\%), S-train (1.7\%) and metro (0.5) while the non-motorized modes take $4.0 \%$ of total inland travel demand.

Fig. 3 presents the travel demand disaggregated on urbanization type in 2015 from LTM. It shows that most of the trips in east take place in urban area while in west, rural area has the highest share.

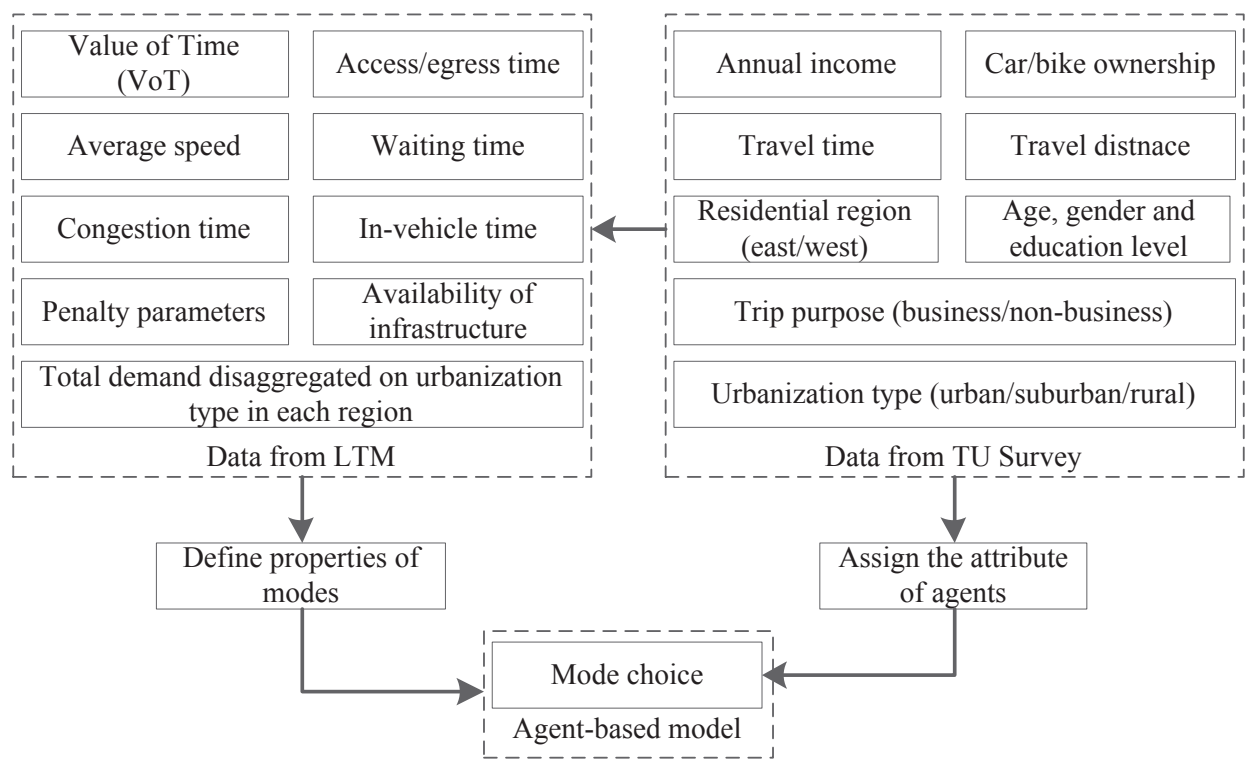

Fig. 1. Data parameters within ABMoS-DK. 


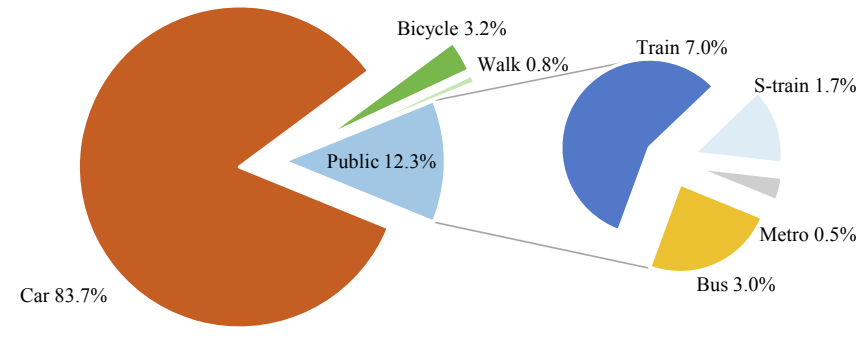

- Car $=$ Bicycle = Walk $=$ Bus $=$ Train S-train " Metro

Fig. 2. Shares of different transport modes in 2015, Million passenger kilometer (LTM).

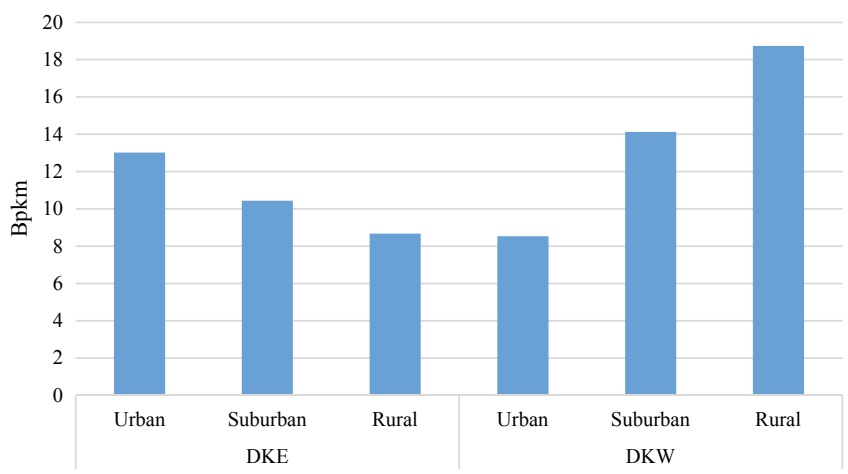

Fig. 3. Travel demand disaggregated on urbanization type in 2015 (LTM).

\subsection{Methodological framework}

The methodological framework is presented in Fig. 4. The base year of the model is 2010 and the model runs until 2050. First, the model reads the TU survey database and parametrize the attributes of heterogeneous agents with socio-economic characteristics and travel demand from 2010 until 2015. For the modeled years after 2015 until 2050 , the majority of agents continue and some agents are generated with the same characteristics from the database randomly to reflect the changes in socio-economic and population synthetics. Since the travel behaviour data is not available for future years, we run a Monte Carlo simulation for generating agents. Then each traveler agent searches for the available mode and derive a utility for each available option based on tangible costs and value of travel time. The agent chooses the mode of transport with the highest utility based on rational choice theory. The model uses rational choice theory for expressing and scoring the direction and importance of the effect of tangible cost or value of travel time (properties of modes) built on the premise of individual utility maximization. ABMoS-DK simulates mobility behaviour of travelers and forecast modal shares endogenously for future years.

In the TU survey, each interviewee has an associated weighting factor that is determined in a way so that the surveyed population synthesizes and reproduces the real Danish population. The 2015 version of TU Survey consists of 134,044 interviews, 23,753 of which were conducted in 2010 (TU Survey, 2018). The yearly records of interviews are defined as number of agents for the years until 2015 and the model generated total of $11,087,601$ agents for future milestone years (i.e., 2020, 2030, 2040 and 2050) including 50 replications. In ABMoS-DK, each agent represents a group of travelers with homogenous socioeconomic characteristics and the heterogeneity of travelers is defined by generating population of agents to simulate that different groups of transport users have specific preferences. The weighting factor is used to specify the number of people represented by each heterogeneous agent. The huge number of agents in the model captures a fine resolution of heterogeneity of socio-economic characteristics. Since the

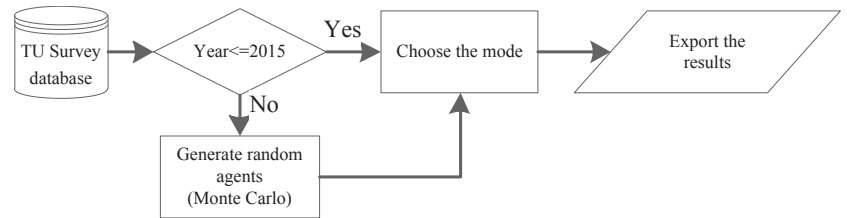

Fig. 4. Methodological framework.

mode choice results from bottom-up travelers' behaviour, the ABM approach will also enable the user to study aggregate properties on desired level. This could help policy makers to analyze the potential of imposing policies in different geographical zones, on specific group of people (e.g., age, income, gender, education level, car ownership) and for certain trip purposes in long-time horizon.

To facilitate the analysis of results and better understand the trends, we have further grouped agents based on region, trip length, urbanization type for place of residence and annual household income. The place of residence is defined as Denmark east and west while the urbanization pattern is Urban (U); Suburban (S) and Rural (R). The classification of trip lengths is: Extra-short (XS) less than or equal to $5 \mathrm{~km}$; Short (S) between 5 and $25 \mathrm{~km}$; Medium (M) between 25 and $50 \mathrm{~km}$; Long (L) more than $50 \mathrm{~km}$. The household income groups are: Very Low (VL) less than $200 \mathrm{kDKK}^{1}$ /year; Low (L) between 200 and 500; Medium (M) between 500 and 800; High (H) more than $800 \mathrm{kDKK} / \mathrm{year}$. The agents look for an appropriate mode of transport in the traffic system to meet annual travel demand. They decide on the preferred mode of transport according to personal attributes and properties of modes through mode choice algorithm. The ABMoS-DK is capable of analyzing behavioural preferences of travelers and understand the factors that explain their rational behaviour. These factors could be changed through policy scenarios to affect their decision and consequently shifting to more sustainable modes.

ABMoS-DK is simulated using AnyLogic multimethod simulation tool developed at Experimental Object Technologies (http://www. xjtek.com) which is a tool for modeling and simulation of complex systems (Borshchev et al., 2000; Borshchev et al., 2002). ABMoS-DK runs on Java platform hosted by AnyLogic hybrid engine.

\subsection{Modes of transport}

The modes of transport are the transport technologies which have various properties and infrastructure defining the technological subsystem. These transport technologies construct the option list to be chosen by transport user agents based on agents' attributes and properties of technologies to meet travel demand. Table 2 shows the availability of infrastructure, maximum constrained length and analytically elaborated equations inspired from LTM and Tattini et al., (2018b) to calculate tangible costs and value of travel time of each mode of transport in ABMoS-DK, categorized as private, non-motorized and public transport.

\subsubsection{Private car}

The infrastructure for driving a private car is defined as having access to car at household level and having driving license for an ordinary passenger car (i.e., category B). However, the passenger of private car is not required to have a driving license. The tangible cost associated with private cars includes tire, maintenance, insurance, ownership tax, parking cost, depreciation cost and other costs represented in Eq. (1) and the annual fuel cost calculated using Eq. (2) all taken from FDM (2017):

\footnotetext{
${ }^{1} 1$ Euro equals 7.447 Danish Kroner (DKK) as of 09 January 2018.
} 
Table 2

The details of each mode.

\begin{tabular}{|c|c|c|c|c|}
\hline & $\begin{array}{l}\text { Availability of } \\
\text { infrastructure }\end{array}$ & $\begin{array}{l}\text { Maximum } \\
\text { length }(\mathrm{km})\end{array}$ & Tangible Cost & $\begin{array}{l}\text { Value of } \\
\text { Travel Time } \\
\text { (VOTT) }\end{array}$ \\
\hline Private car & All zones & Unlimited & Eq. (1) & Eq. (3) \\
\hline Walk & All zones & 15 & N/A & Eq. (5) \\
\hline Bike & All zones & 25 & Eq. (6) & Eq. (7) \\
\hline Bus & All zones & Unlimited & Ticket price & Eq. (8) \\
\hline Train & All zones & Unlimited & Ticket price & Eq. (8) \\
\hline S-Train & $\begin{array}{l}\text { Greater } \\
\text { Copenhagen Area }\end{array}$ & 63 & Ticket price & Eq. (8) \\
\hline Metro & Copenhagen City & 14.2 & Ticket price & Eq. (8) \\
\hline
\end{tabular}

$$
\begin{aligned}
C_{p c}^{\text {Tangible }}= & L_{p c}^{\text {Trip }} \times\left(C_{a}^{\text {Fuel }}+C_{a}^{\text {Tire }}+C_{a}^{\text {Maintenance }}+C_{a}^{\text {Insurance }}+C_{a}^{\text {Tax }}+C_{a}^{\text {Dep }}\right. \\
& \left.+C_{a}^{\text {Other }}\right) / M_{a} \\
C_{a}^{\text {Fuel }}= & M_{a, p c} \times F P / E F_{p c}
\end{aligned}
$$

where $C$ denotes cost (DKK), $p c$ denotes private mode of transport, $a$ stands for annual, $M_{a}$ denotes annual mileage (km/year), $F P$ denotes the fuel price (DKK/liter) and FE represents fuel economy (km/liter). The value of travel time for private car is calculated based on value of time (as a mediator parameter to monetize travel time) which changes across households' income and trip purpose using Eq. (3) while Eq. (4) calculates the in-vehicle time of the trip:

$$
C_{p c}^{V O T T}=V o T_{I C, T P} \times\left(T_{p c}^{\text {InVehicle }}+T_{p c, U T}^{\text {Congestion }} \times \text { Penalty } \text { Congestion }^{\text {Cot }}\right)
$$

$T_{p c}^{\text {InVehicle }}=L_{p c}^{\text {Trip }} / S_{p c, U T}^{\text {Average }}$

VoT is the value of time changing across households' income class (IC) and trip purpose (TP) (e.g., business vs. non-business trip) expressed in the unit of DKK/min. T represents travel time (in minute) and includes in-vehicle and congestion time. The congestion time varies across urbanization type and congestion penalty varies across trip purpose (TP) both taken from LTM. Congestion penalty represent the inconveniences associated with congested driving time. $L$ stands for trip length and $S$ is the average speed changing across urbanization type (UT). VoT and average speed for each mode across urbanization type are taken from LTM. In this model it is assumed that in urban areas during rush hour, the speed of private cars decreases by $30 \%$ while the congestion time of private cars increases by $30 \%$ calculated from LTM. Moreover, if the agent is a member of car sharing scheme, the cost is equal to the duration of trip multiplied by cost of car sharing per minute plus the value of travel time associated with driving. The algorithm uses the same VOTT associated with private cars for passengers in a car sharing scheme.

\subsubsection{Non-motorized transport (NMT)}

The non-motorized modes of transport include walking and bicycling which are options only available for extra short and short trips. There is no tangible cost associated with walking. As suggested by Hammadou and Papaix (2015), ageing largely influences walking activities. Therefore, if the agent is under the age 18 or over age 65 , the average speed of walking across all urbanization types is decreased by $20 \%$. The value of travel time of walking is calculated based on value of time (as a mediator parameter to monetize travel time) which changes across households' income and trip purpose using Eq. (5):

$C_{w}^{\text {VOTT }}=V O T_{I C, T P} \times\left(L_{w}^{\text {Trip }} / S_{w, U T, \text { age }}^{\text {Average }}\right)$

where $w$ stands for walk and $S$ is the average speed of walk changing across urbanization type (UT) and age of traveler.

Eq. (6) calculates the tangible cost of cycling. If the agent owns an electric bike, the electricity price and maintenance cost will constitute the tangible cost. For electric bikes, on average, a $250-\mathrm{W}$ battery will provide a range of $55 \mathrm{~km}$, and the cost of charging is $5.25 \mathrm{DKK} / \mathrm{kWh}$ (Mobycon, 2014) so full battery charge costs 1.32 DKK and one kilometer by electric biking costs 0.024 DKK. Therefore, trips using electric bikes are constrained to $55 \mathrm{~km}$ and the tangible costs of cycling are given as:

$C_{b}^{\text {Tangible }}=L_{b}^{\text {Trip }} \times\left(C_{b}^{\text {Maintenance }}+C_{i f e-\text { bike }}^{\text {Electricity }} \times 0.024\right)$

where, $b$ denotes bicycle. The maintenance cost of cycling is taken from triangular probability distribution (i.e., $\min =0.01, \quad \max =1$, mode $=0.5) \mathrm{DKK} / \mathrm{km}$. In ABMoS-DK agents between 14 and 50 years old who own a bicycle may ride for up to $25 \mathrm{~km}$, cycling is only an option for agents younger than 75 years and agents within the other age classes will have $20 \%$ higher VOTT associated with riding a bicycle determined from LTM. The VOTT of cycling is calculated using Eq. (7).

$C_{b}^{\text {VOTT }}=V_{\text {OOT }} T_{I C, T P} \times\left(L_{b}^{\text {Trip }} / S_{b, U T, \text { age }}^{\text {Average }}\right)$

\subsubsection{Public transport}

The public modes include bus, train, S-train (urban-suburban railways) and metro. The tangible cost of each mode of public transport equals to ticket cost, which is related to trip length and provided exogenously while the VOTT of each public modes are calculated endogenously using Eq. (8):

$$
\begin{aligned}
C_{p t}^{\text {VOTT }}= & V_{\text {o }} T_{I C, T P} \times\left(T_{p t}^{\text {InVehicle }}+\left(T_{p t, U T}^{\text {Wait }} \times \text { Penalty } y_{p t}^{\text {Wait }}\right)\right. \\
& \left.+\left(T_{p t, U T}^{A C C / E G R} \times \text { Penalty }{ }_{p t}^{A C C / E G R}\right)\right)
\end{aligned}
$$

where $p t$ stands for public transport (i.e., bus, train, S-train and metro), penalty parameters for waiting (Wait) time and access/egress time (ACC/EGR) is constant and equal to 1.5 taken from LTM and represent the inconveniences associated with waiting and access egress time. The in-vehicle time is calculated using Eq. (9).

$T_{p t}^{\text {InVehicle }}=L_{p t}^{\text {Trip }} / S_{p t, U T}^{\text {Average }}$

It is assumed that the agent could study or work while commuting in public transport (Malokin et al., 2015) introduced as intangible benefit. Therefore, for educated agents with medium or long commuting distance, there is no in-vehicle time associated with the value of travel time. Eq. (10) calculates the total cost across the consumer groups and for each 7 alternative modes in the model.

$C_{m}^{\text {Total }}=\gamma_{1} \times C_{m}^{\text {Tangible }}+\gamma_{2} \times C_{m}^{\text {VOTT }}$

where $\gamma$ parameter defines the direction and importance of the effect of tangible cost and value of travel time attribute on the utility of alternative estimated based on travel budget and travel time across income groups and later calibrated.

\subsection{Mode choice}

Fig. 5 presents the flowchart of ABMoS-DK to determine the mode of transport based on the traveler's personal attributes, expectations, properties of modes and availability of infrastructure. The traveler's heterogeneity is incorporated to take into account that different users have specific preferences affecting modal choice. Each agent individually assesses its situation and makes decision on the basis of a set of rules (Bonabeau, 2002).

Each agent derives a utility for each alternative mode calculated based on the observed factors of the trip i.e., tangible costs and value of travel time, and the traveler chooses the mode with the highest utility. The derived utility of each mode also depends on the socio-economic and behavioural characteristics of the households (urbanization pattern, income level and value of time). The expectations of travelers are adopted according to the budget (travel money budget) and time (travel time budget) they are willing and able to spend to meet travel demand 


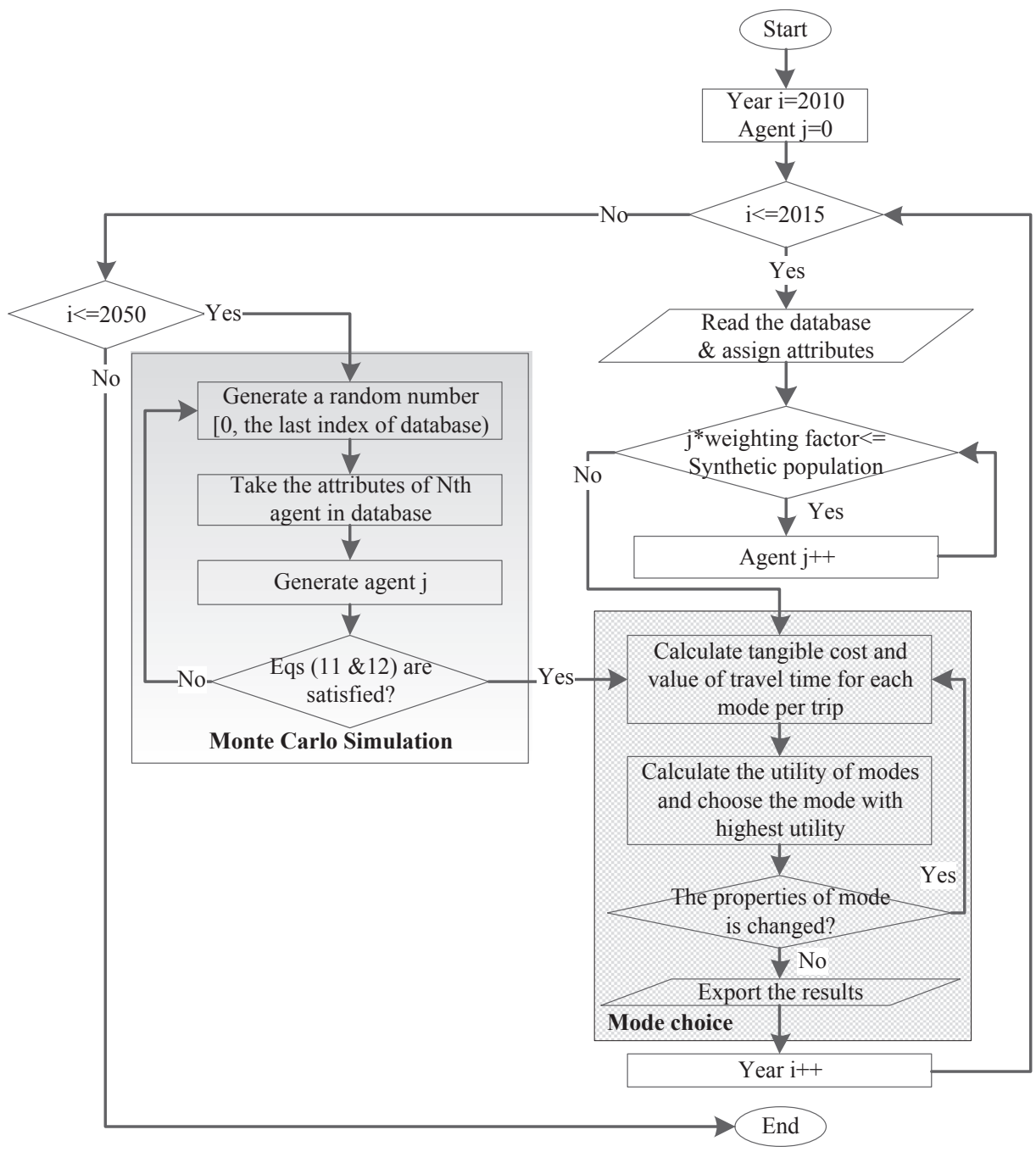

Fig. 5. Flowchart of ABMoS-DK.

across income groups. For instance, the competition between faster and more expansive modes (e.g. car) with slower but cheaper modes (e.g. bus or rail) is ensured by evaluating the derived utilities of each mode based on the travel budget and travel time representing expectation of travelers. However, agents have memory and in case that the derived utilities of alternative choices are equal, the agent continues to habitually repeat previous behaviour (taken from TU survey).

\subsection{Interactions}

Within ABMoS-DK, traveler agents make decision independently and neither communicate with others nor learn from other agents. Therefore, there is no direct interaction between traveler agents. However, a large number of agents act autonomously at micro level and choose a specific mode of transport affecting some properties of the available modes in the network. The more agents using private car in a certain zone emerges congestion which affects speed, in-vehicle and congestion time in Eq. (3). The more agents choosing a certain mode of public transport in specific zone, increases the waiting time in Eq. (8). Moreover, by approaching saturation capacity of the modes, the inconveniences associated with congestion and waiting time increases. Agents who enter the mode choice, observe the situation of the traffic system and sense the properties of modes emerged from action of other agents. Hence, the derived utility of mode changes, which might affect the mode choice decision. The interactions are mediated by the system dynamics emerged from the action of travelers in the traffic system such as "SimCo" framework developed by Adelt et al. (2018) and ABM package of TRANSIMS (Bonabeau, 2002). Therefore, the modeling process simulates the interaction between travelers (agents) and the network.

\subsection{Generating agents}

The TU survey contains data for the model years 2010-2015. For the years 2016-2050, agents are randomly generated and their associated characteristics are taken from the TU survey database i.e., ABMoS-DK generates a random number $N \in$ $\left[0\right.$, the Last IndexOfDatabase) and takes the attributes of $\mathrm{N}^{\text {th }}$ agent. Two criteria i.e. forecast of travel demand split by urbanization area and projection of synthetic population are defined to stop random agent generation. This loop iterates until Eq. (11) and Eq. (12) are satisfied:

$\sum_{j} L_{U T, j}^{\text {Trip }} \times W_{j}^{\text {Factor }} \times N^{\text {Days }} \geq D_{i}^{U T}$

$L$ stands for trip length originated in one of the urbanization types (i.e., east/west, urban, suburban or rural), $j$ is the representing agent, $W$ is a weighting factor taken from TU survey representing the number of people with the homogenous characteristics and travel demand in the entire Danish population. $N$ is the number of days in a year (varies across working and non-working days). The right hand side of the Eq. (11) is the forecast of travel demand in year $i$ taken from LTM disaggregated on urbanization type. 


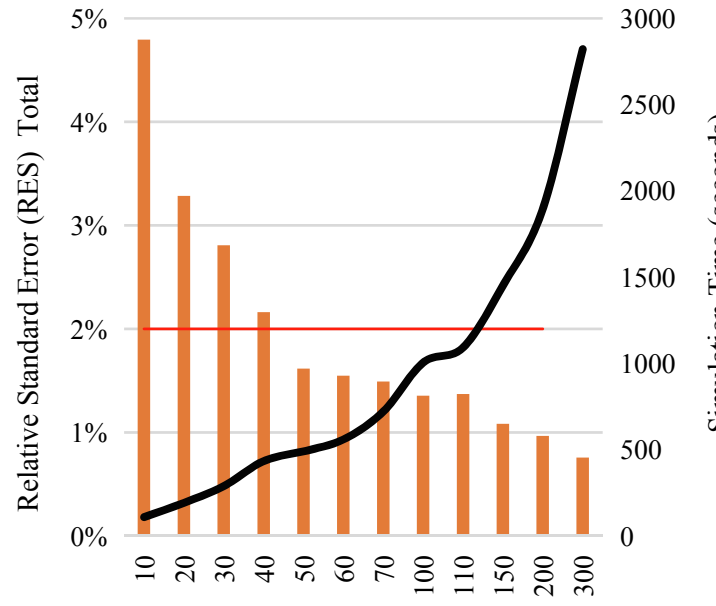

Replications

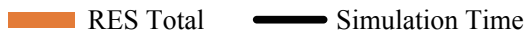

Fig. 6. Required number of replications.

The annual historical demographic data and projection of synthetic population of country is taken from statistics Denmark out to the year 2050 (Statistics Denmark, n.d.). The second criteria for stopping random agent generation intends to reproduce the annual projection of synthetic population using Eq. (12):

$\sum_{\text {year }} W^{\text {Factor }} \cong P^{i}$

where $\mathrm{P}^{\mathrm{i}}$ represents the synthetic population projections by statistics Denmark. In other words, the agents are generated using a Monte Carlo simulation such that the aggregation of demand in each urbanization area together with population synthesis matches the LTM demand and demographic data respectively.

Several experiments were completed to determine how the number of Monte Carlo replications affected the confidence level. Fig. 6 represents the Relative Standard Error (RSE) calculated using Eq. (13). Increasing the number of replications decreases the RSE while increasing the simulation time. The trial showed that 50 replications (generating approximately 11 million agents) results in a RSE well below $2 \%$ with a simulation time of $434 \mathrm{~s}$ (Fig. 6). Therefore, in order to resolve the problem of uncertainty raised from random agent's generation, we run the model for years 2015-2050 with 50 replications.
$R S E_{\text {Total }}=\sqrt{\sum_{\text {AllModes }}\left(\frac{S E}{\text { meanValue }}\right)^{2}}$

Fifty replications is in line with similar Monte Carlo-based studies of this nature. For instance, Qu and Zhou (2017) executed 10 iterations to reduce sampling errors. Boateng and Awuah-Offei (2017) run the Monte Carlo simulation 20 times with 20,000 agents. Ahanchian and Biona (2017) and Sopha et al. (2011) performed 30 replications.

\subsection{Calibration and validation}

ABMoS-DK reads the database and assigns the attributes of traveler (such as age, gender, education level, car/bike ownership, membership in car sharing scheme, driver's license ownership and income) to agent and calculate trip characteristics (such as departure time and day, urbanization type of origin and destination, trip purpose and trip length). The properties of modes (such as availability of infrastructure, average speed, average access/egress time, average waiting time and average in-vehicle time in each urbanization type) taken from LTM are assigned to modes. The agents with specific travel demand follow the mode choice algorithm to decide on the mode of transport. The calibration process accomplished by adjusting the number of working days and non-working days in the year, the congestion during rush hours, speed, access/egress, waiting time and the importance of the effect of tangible cost and value of travel time to reproduce the historical modal share in 2010. The calibrated and adjusted model was run to check the ability of reproducing the historical modal share in 2015 from LTM. The left hand side of Fig. 7 shows the results of modal shares compared to the historical data from LTM in 2010 while the right hand side shows the results of modal shares compared to the historical data from LTM in 2015. The calibrated model is then run until the last year of simulation in order to forecast the modal shares.

\subsection{Scenario definition}

One reference scenario and four alternative scenarios are developed based on recent policy debates adopted from (Mathiesen, and Kappel, 2013) and tested to determine the effect on modal shift, and shift away from private cars in particular. All scenarios include the current expansion of existing Copenhagen Metro, which includes $15.5 \mathrm{~km}$ of new underground railway and 17 new stations. The new city ring line is planned to be opened in 2020 .

\subsubsection{Business as usual (BAU)}

The BAU scenario represents a continuation of current conditions based on the TU and LTM. The business as usual serves as the base (reference) scenario in our study.

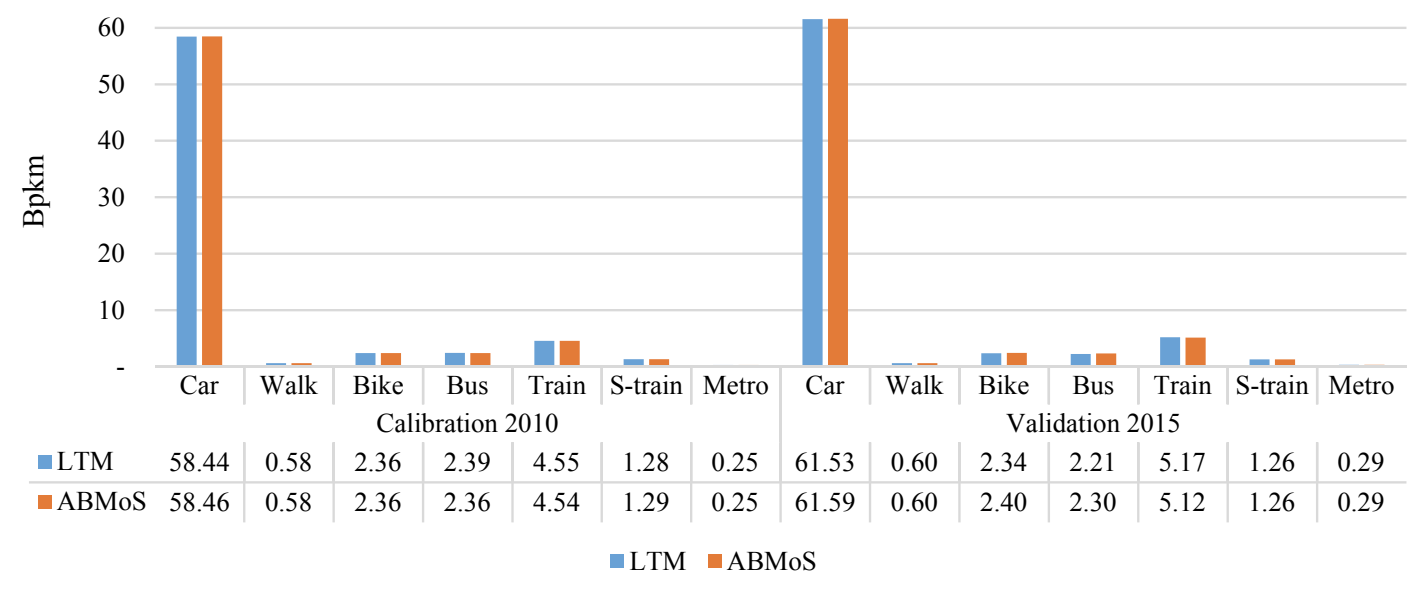

Fig. 7. Calibration and validation. 


\subsubsection{Expansion of public infrastructure (EPI)}

Encouraging the travelers to shift to more sustainable modes could be promoted by increasing the utility of public modes (e.g., decreasing value of travel time: access/egress and waiting time) provided that the infrastructure is available in the area. In this scenario, we analyze the effect of developing metro in the Denmark west urban areas (i.e., Aarhus, Aalborg and Odense). S-train railways would also be available in western urban and suburban areas by 2025 , and the frequency of all trains and buses is increased by $10 \%$ with respect to reference scenario. Based on the timetable of public modes, some modes are not available in some areas during nighttime. In this scenario, however, there is public transport available every two hours.

\subsubsection{Incentives for sustainable modes (ISM)}

Decreasing the tangible cost of more sustainable modes increases the utility of these modes and might affect the mode choice decision. ISM examines the impact of decreasing public transport ticket price by $20 \%$ in 2025 . Additionally, free parking is assumed to be available for train and S-train users, thus eliminating the access and egress time to public transport. The access and egress stage (parking a private car) is added to the primary mode of the trip. Finally, in this scenario, all bicycles are assumed to be electric with free recharging of the battery, thereby extending the maximum trip length to $55 \mathrm{~km}$.

\subsubsection{Disincentives for private cars (DPC)}

Increasing the tangible cost of private cars decreases the utility of this mode. DPC examines increasing the fuel tax by $50 \%$; increasing registration and annual ownership tax of a fossil fuel dependent vehicle by $50 \%$; doubling the parking cost, and collecting toll on vehicles coming into Copenhagen (30 DKK per trip irrespective of trip length during weekdays from 6 am to $6 \mathrm{pm}$ ).

\subsubsection{Combination of all scenarios (COM)}

A set of alternative "pull" and "push" scenarios acting independently of others are developed to evaluate the effect of importance of tangible cost and value of travel time on mode choice. In this scenario integration of above policy instruments are tested to achieve greater performance from the overall strategy (May et al., 2006).

\section{Results}

ABMoS-DK determines the maximum shift potential in the inland passenger transport sector of Denmark endogenously from the perspective of consumers while interacting with the network. The analysis shows that the total demand in 2010 was $69.845 \mathrm{Bpkm}$ which would increase to $91.375 \mathrm{Bpkm}$ in 2050 representing an increase of around $30.8 \%$ compared to base year under reference scenario. The Monte Carlo simulation was performed 50 times to calculate the absolute value of the modal split (Billion-passenger-kilometer) together with the standard deviation. However, in order to have a comparable analysis with respect to effectiveness of scenarios, the results in this section present the percentage of modal choice. Due to the definition of scenarios, modal shift takes place from 2030 onwards.
Table 3 shows the percentage change of modal shares across scenarios in 2050 compared with the reference scenario in the whole country. The contribution of scenario which encourage the travelers to reduce car ridership and shift to other modes, demonstrates the effectiveness of scenario. To provide an overview regarding the effectiveness of scenarios, the changes with more than 10 percent are colored. The green color represents changes towards sustainability while the pink color represents the deterioration of the situation.

The results of the analysis in BAU (reference) scenario show that in 2050, the demand for car ridership increases by $31.7 \%$ with respect to the base year (2010), because of its availability almost everywhere, often associated with higher travel speed and in some cases, with lower total costs. The demand for metro increases by $135.2 \%$ saturating the current and the new city ring infrastructure due to the level of service. Train ridership also increases by $69.7 \%$ due to relatively higher speed and comparative lower cost for long trips. Since, there is no tangible cost for walking, the demand in this mode increases by $25.0 \%$ while, biking increases slightly by $0.6 \%$. The results show that S-train and bus ridership decrease by $4.3 \%$ and $25.3 \%$ respectively which might be substituted by other modes due to comparative lower level of service.

The expansion of public infrastructure scenario is to analyze the impact of developing the infrastructure of public transit on modal choice. The results of this scenario show that expansion of metro and Strain infrastructure provide opportunities to increase their ridership by $594.7 \%$ and $110.9 \%$ in 2050 compared to the reference scenario respectively. Moreover, increasing the frequency of public transit decreases the waiting time thus the value of travel time of public modes decrease resulting in $14.9 \%$ and $8.0 \%$ increase of ridership for bus and train respectively. Biking increases slightly by $1.1 \%$ while the use of private cars and walking are decreased by $7.1 \%$ and $40.6 \%$ respectively. This might be due to better performance and comparative advantages of public transport which encourage the travelers to shift to these modes.

The result of incentives for sustainable modes scenario shows that incentivizing the use of public transit and electric bikes increase the share of metro by $554.6 \%$ followed by S-train ( $422.5 \%)$, bike $(66.4 \%)$ and train $(61.8 \%)$ compared to the reference scenario in 2050 . However, the bus ridership, private cars and walking experience decrease of $1.0 \%, 19.0 \%$ and $28.7 \%$ respectively, which are obviously covered by other public modes. The incentives for sustainable modes scenario which decreases the tangible cost of public transit and electric bikes, seems to be more successful with regards to reducing car ridership compared to expansion of public infrastructure scenario by decreases the value of travel time. This might be due to the perception of travelers and the importance level of tangible cost over value of travel time. These two scenarios could be categorized as pull policy.

The results of disincentives for private cars scenario show that increasing the cost of private cars as a "push" policy discourages the use of cars by $29.8 \%$ compared to the reference scenario in 2050 . Therefore, metro increases by $772.4 \%$ followed by S-train $(282.9 \%)$, bus $(234.5 \%)$, bike $(175.8 \%)$ and walk $(1.5 \%)$ respectively. The higher shift potential of car use under disincentives for private cars scenario compared to previous scenarios might be due to the fact that travelers prefer to avoid paying more tangible cost rather than receiving

Table 3

The change of modal shares across scenarios in 2050 compared with the reference scenario.

\begin{tabular}{|c|c|c|c|c|c|c|c|c|}
\hline & w.r.t BAU & Private & Walk & Bike & Bus & Train & S-train & Metro \\
\hline Business as Usual (BAU) & in 2010 & $31.70 \%$ & $25.00 \%$ & $0.60 \%$ & $-25.30 \%$ & $69.70 \%$ & $-4.30 \%$ & $135.20 \%$ \\
\hline Expansion of Public Infrastructure (EPI) & in 2050 & $-7.10 \%$ & $-40.60 \%$ & $1.10 \%$ & $14.90 \%$ & $8.00 \%$ & $110.90 \%$ & $594.70 \%$ \\
\hline Incentives for Sustainable Modes (ISM) & in 2050 & $-19.00 \%$ & $-28.70 \%$ & $66.40 \%$ & $-1.00 \%$ & $61.80 \%$ & $422.50 \%$ & $554.60 \%$ \\
\hline Disincentives for Private Cars (DPC) & in 2050 & $-29.80 \%$ & $1.50 \%$ & $175.80 \%$ & $234.50 \%$ & $85.80 \%$ & $282.90 \%$ & $772.40 \%$ \\
\hline Combination of all scenarios (COM) & in 2050 & $-49.30 \%$ & $-53.70 \%$ & $464.80 \%$ & $158.10 \%$ & $112.40 \%$ & $853.20 \%$ & $906.10 \%$ \\
\hline
\end{tabular}



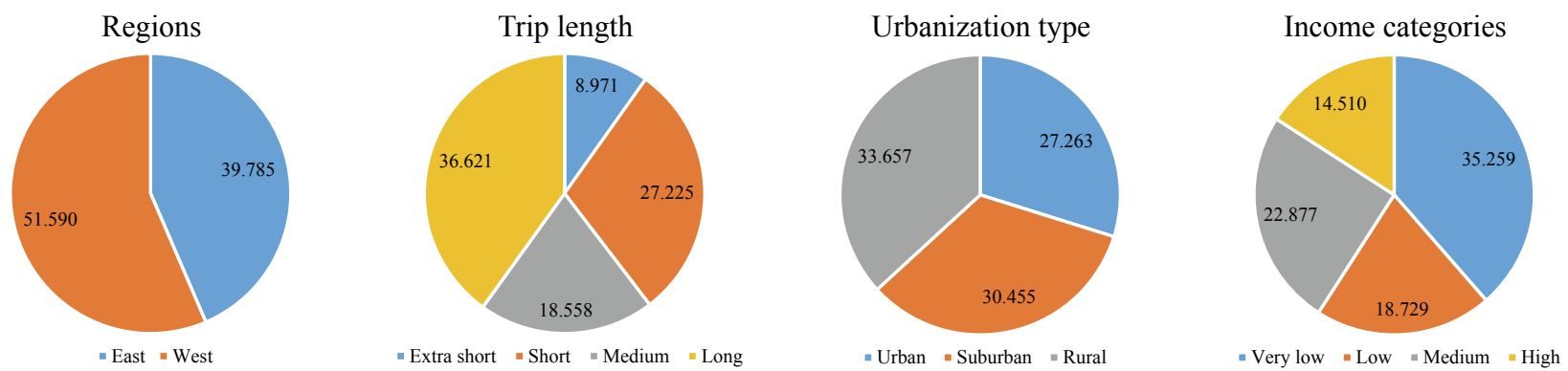

Fig. 8. Absolute value of travel demand grouped based on regions, trip length, urbanization type and income in 2050 (Bpkm).

subsidies or spending less time.

A combination of "pull" and "push" scenarios simultaneously might provide the most promising results with regards to decrease of car use representing $49.3 \%$ car use reduction with respect to the reference scenario in 2050. The results show that the combination of scenarios increases the use of metro by $906.1 \%$, followed by S-train $(853.2 \%)$, bike $(464.8 \%)$, bus $(158.1 \%)$ and train $(112.4 \%)$ respectively. In this scenario, walking decreases by $53.7 \%$ which is due to comparative advantages of other modes over walk.

The bottom-up agent-based modeling approach is flexible with regards to aggregating the simulated behaviour of consumer groups. By understanding the modal shift potential of different groups, the policies could be prioritized targeting group of agents. Fig. 8 demonstrates the absolute value of travel demand grouped based on regions, trip length, urbanization type and income in 2050 (Bpkm). Aggregating the results based on regions show that the total demand in west $(51.590 \mathrm{Bpkm})$ is higher than east $(39.785 \mathrm{Bpkm})$ probably due to bigger area, less concentration of residential places and consequently longer travel distances. Summing the results based on trip length indicates that the long distance trips have the highest demand equal to $36.621 \mathrm{Bpkm}$ followed by short distance 27.225 , medium distance 18.558 and X-short distance trips $8.971 \mathrm{Bpkm}$. Aggregating the results based on urbanization type of residential location depicts that the total demand in rural area is 33.657 Bpkm followed by suburban (30.455) and urban are (27.263) which might be due to longer distances in rural areas. Aggregating the results based on annual income illustrates that the total travel demand has the highest value in very low income group (32.259 Bpkm) followed by medium (22.877), low (18.729) and high (14.51) Bpkm.

The effectiveness of scenarios for each of the above categories are further analyzed below. The scenarios change the share of modes and do not affect the total demand in each category. Table 4 compares the modal share potential across alternative scenarios in east and west in
2050. Private cars have the highest share of regional demand in the reference scenario representing $81.4 \%$ in east and $86.4 \%$ in west. However, the results show that implementing strategies in east is more effective at reducing car use compared to west probably due to shorter trip demands and better performance of public transport. By implementing the combination of scenarios, the share of private cars could be reduced to $32.1 \%$ and $50.9 \%$ in east and west respectively. In the reference scenario, the share of bicycling is $2.9 \%$ in east and $2.4 \%$ in west. Apparently, expanding the public infrastructure affects bicycling slightly while the incentives for sustainable modes increase the share of bicycling to $4.4 \%$ and $4.3 \%$ in east and west respectively. Increasing the cost of private cars encourages travelers to use bike and increase the share of this mode to $6.8 \%$ and $7.4 \%$ in east and west respectively. Combination of scenarios is more effective for increasing the share of bicycling in western region (16.0\%) compared to eastern region $(12.9 \%)$ perhaps because of more expensive cost of private cars, agents prefer to shift to other mode. However, since the public infrastructure could not cover some travel demands is west, agents shift to bike. Walking has slight share compared to other modes in both regions. Although in some scenarios the share of walking reduces with respect to the reference scenario, bikes compensate this reduction and the total share of non-motorized modes provide promising results. The S-train and metro infrastructure are not available in west under business as usual, incentives for sustainable modes and disincentives for private cars scenarios. However, by expanding the infrastructure, S-train and metro could be responsible to accommodate $2.6 \%$ and $1.3 \%$ of total demand in the western region respectively and by implementing the combination of scenarios these values could reach to $6.1 \%$ and $1.7 \%$ respectively. In the reference scenario, the share of all public modes is $15.2 \%$ and $10.1 \%$ in east and west. The results show that the simulated scenarios contribute in increasing the share of public modes in east more than west.

Table 4

Comparing the modal share potential across scenarios in 2050 (east vs. west) in percentage.

\begin{tabular}{lc|c|c|c|c|c|c|c|c|c}
\hline & \multicolumn{5}{c|}{ Denmark east } & \multicolumn{5}{c}{ Denmark west } \\
\cline { 2 - 12 } & BAU & EPI & ISM & DPC & COM & BAU & EPI & ISM & DPC & COM \\
\hline Private & $\mathbf{8 1 . 4}$ & $\mathbf{7 3 . 2}$ & $\mathbf{5 5 . 9}$ & $\mathbf{4 6 . 5}$ & $\mathbf{3 2 . 1}$ & $\mathbf{8 6 . 4}$ & $\mathbf{8 2 . 2}$ & $\mathbf{7 7 . 8}$ & $\mathbf{6 8 . 9}$ & $\mathbf{5 0 . 9}$ \\
\hline Sum Non-motorized & $\mathbf{3 . 4}$ & $\mathbf{3 . 1}$ & $\mathbf{4 . 8}$ & $\mathbf{7 . 2}$ & $\mathbf{1 3 . 2}$ & $\mathbf{3 . 4}$ & $\mathbf{3 . 1}$ & $\mathbf{5}$ & $\mathbf{8 . 6}$ & $\mathbf{1 6 . 5}$ \\
$\quad$ Walk & 0.5 & 0.3 & 0.4 & 0.4 & 0.3 & 1.1 & 0.6 & 0.7 & 1.1 & 0.4 \\
Bike & 2.9 & 2.8 & 4.4 & 6.8 & 12.9 & 2.4 & 2.5 & 4.3 & 7.4 & 16 \\
\hline Sum Public & $\mathbf{1 5 . 2}$ & $\mathbf{2 3 . 7}$ & $\mathbf{3 9 . 3}$ & $\mathbf{4 6 . 3}$ & $\mathbf{5 4 . 7}$ & $\mathbf{1 0 . 1}$ & $\mathbf{1 4 . 7}$ & $\mathbf{1 7 . 2}$ & $\mathbf{2 2 . 6}$ & $\mathbf{3 2 . 6}$ \\
Bus & 2.2 & 2.4 & 1.6 & 7 & 4.9 & 1.7 & 2.1 & 2.2 & 6.1 & 5.1 \\
Train & 8.4 & 9.6 & 11.9 & 14.6 & 15.5 & 8.4 & 8.7 & 15 & 16.5 & 19.7 \\
S-train & 3.1 & 3.2 & 16.2 & 11.9 & 21.6 & 0 & 2.6 & 0 & 0 & 6.1 \\
Metro & 1.5 & 8.5 & 9.7 & 12.9 & 12.7 & 0 & 1.3 & 0 & 0 & 1.7 \\
\hline
\end{tabular}

The darker color represents better effect towards decarbonization. 
Table 5

Comparing the modal share potential across scenarios in 2050 based on trip length categories (percentage).

\begin{tabular}{|c|c|c|c|c|c|c|c|c|c|c|c|c|c|c|c|c|c|c|c|c|}
\hline & \multicolumn{5}{|c|}{ Extra short $\leq 5 \mathrm{~km}$} & \multicolumn{5}{|c|}{$5 \mathrm{~km}<$ Short $\leq 25 \mathrm{~km}$} & \multicolumn{5}{|c|}{$25 \mathrm{~km}<$ Medium $\leq 50 \mathrm{~km}$} & \multicolumn{5}{|c|}{$50 \mathrm{~km}<$ Long } \\
\hline & BAU & EPI & ISM & DPC & $\mathrm{COM}$ & BAU & EPI & ISM & DPC & $\mathrm{COM}$ & BAU & EPI & ISM & DPC & $\mathrm{COM}$ & BAU & EPI & ISM & $\mathrm{DPC}$ & COM \\
\hline Private & 64 & 62.8 & 62.1 & 46.9 & 38.9 & 96.3 & 87.4 & 85.3 & 71.4 & 56.2 & 91.9 & 87.7 & 67.7 & 67.2 & 39.3 & 76.3 & 70.5 & $\mathbf{5 7 . 5}$ & 48.8 & 35.4 \\
\hline $\begin{array}{l}\text { Sum Non- } \\
\text { motorized }\end{array}$ & 27.6 & 25.8 & 33.5 & 37.5 & 53.6 & 1.9 & 1.6 & 2.9 & 12.4 & 21.8 & 0.5 & 0.5 & 3.3 & 3 & 14.9 & 0.1 & $\mathbf{0}$ & 0.1 & $\mathbf{0}$ & 0.6 \\
\hline Walk & 6.9 & 4.8 & 5.8 & 8.2 & 3.7 & 0.3 & 0 & 0 & 0 & 0 & 0 & 0 & 0 & 0 & 0 & 0 & 0 & 0 & 0 & 0 \\
\hline Bike & 20.6 & 21 & 27.7 & 29.3 & 49.9 & 1.6 & 1.6 & 2.9 & 12.4 & 21.8 & 0.5 & 0.5 & 3.3 & 3 & 14.9 & 0 & 0 & 0.1 & 0 & 0.6 \\
\hline Sum Public & 8.4 & 11.4 & 4.5 & 15.6 & 7.5 & 1.8 & 11 & 11.8 & 16.2 & 22 & 7.5 & 11.8 & 29 & 29.8 & 45.7 & 23.6 & 29.5 & 42.4 & 51.2 & 64 \\
\hline Bus & 6.9 & 4.3 & 1.6 & 5.5 & 0.7 & 0 & 0 & 0 & 0.2 & 0 & 0.1 & 0.1 & 0 & 2.7 & 0.2 & 3 & 4.4 & 4.4 & 13.2 & 12.1 \\
\hline Train & 0 & 0 & 0 & 0 & 0 & 0 & 0 & 0 & 0 & 0 & 6.1 & 7 & 6.1 & 12.8 & 9.8 & 17.9 & 19.2 & 31 & 32.6 & 39.7 \\
\hline S-train & 0.9 & 1.5 & 0 & 0.7 & 0 & 0.1 & 0.1 & 0.2 & 1.9 & 4.8 & 0.9 & 1.3 & 20.5 & 11.8 & 32.4 & 2.6 & 6 & 7.1 & 5.3 & 12.1 \\
\hline Metro & 0.6 & 5.6 & 2.9 & 9.4 & 6.7 & 1.7 & 10.9 & 11.5 & 14.1 & 17.2 & 0.4 & 3.4 & 2.4 & 2.5 & 3.4 & 0 & 0 & 0 & 0 & 0 \\
\hline
\end{tabular}

The darker color represents better effect towards decarbonization.

Table 5 shows the modal share potential across scenarios in 2050 based on four trip length categories. The results show that the simulated scenarios are more effective for reducing car use in long trips followed by extra short, medium and short trips and the highest shift potential could be achieved by targeting long distance trips. However, the scenarios are more effective to increase the total share of non-motorized modes in extra short trips followed by short and medium trips. Train is only a choice for medium and long trips while metro is not a choice for long trips. These scenarios are more effective to increase the total share of public modes in long distances followed by medium, short and extra short trips.

Table 6 compares the modal share potential across scenarios in 2050 in urban, suburban and rural areas. Metro is only a choice in Copenhagen area, while S-train is not available in rural areas. Therefore, private cars are responsible for the majority of trips in rural area. The scenarios are more effective to reduce the share of car use in urban area followed by suburban and rural area. This is due to longer trips and limited availability of public transit in rural areas. In urban and suburban areas, the public transits play a significant role as substitute for car use while in rural areas, bicycling becomes an important mode of transport in lieu of cars.

Table 7 compares the modal share potential across scenarios in 2050 aggregated based on four income groups. The results show the lower the income, the higher the tendency to use non-motorized modes of transport. Increasing the cost of cars is more effective to reduce car use in very low income group. Combined with expanding the public infrastructure and incentivizing sustainable modes the share of private cars in low income category reduces to $22.8 \%$. Wealthier people prefer to spend less time in transport modes so they choose the faster but more expensive modes of transport. Bus accommodates $3.9 \%$ of total demand for very low income category and the popularity of this mode decreases by increasing the income. Metro has almost the same share among the income categories while train and S-train are preferred by those in the medium and high income categories probably for commuting to work.

\section{Discussion}

\subsection{Methodology insights}

The model framework adopted for this study has several advantages compared to other methods for evaluating modal shift potential with systematic approach. First, ABMoS-DK is capable of evaluating the effect on modal shift from a wide range of policies, e.g. involving the level of service of the modes, consumers' expectation, support schemes to public transport and disincentives to the use of private car. Second, ABMoS-DK is flexible with regards to the level of aggregation. Third, ABMoS-DK is scalable, as it allows the evaluation of modal shares for a smaller portion of the entire system represented. Fourth, it is robust, as it provides consistent results that closely match those of the LTM. Finally, it is fast, taking approximately $15 \mathrm{~min}$ to assess modal shares for scenarios developed.

However, our approach also has some limitations. Due to lack of data, agents decide on mode of transport independently and they do not communicate and do not learn from others while there is an interaction

Table 6

Comparing the modal share potential across scenarios in 2050 based on urbanization type (percentage).

\begin{tabular}{|c|c|c|c|c|c|c|c|c|c|c|c|c|c|c|c|}
\hline & \multicolumn{5}{|c|}{ Urban } & \multicolumn{5}{|c|}{ Suburban } & \multicolumn{5}{|c|}{ Rural } \\
\hline & BAU & EPI & ISM & DPC & $\mathrm{COM}$ & BAU & EPI & ISM & DPC & $\mathrm{COM}$ & BAU & EPI & ISM & DPC & $\mathrm{COM}$ \\
\hline Private & 68.7 & 55.6 & 42.7 & 34.4 & 19.9 & 84.9 & 79.3 & 63.4 & 54.7 & 35 & 96.3 & 95.7 & 93.3 & 82.7 & 67.6 \\
\hline $\begin{array}{l}\text { Sum Non- } \\
\text { motorized }\end{array}$ & 4.1 & 3.5 & 4.8 & 8.3 & 10.9 & 3.5 & 3.3 & 4.8 & 7 & 10.5 & 2.8 & 2.5 & 5 & 8.7 & 22.5 \\
\hline Walk & 0.5 & 0.1 & 0.7 & 0.4 & 0.1 & 0.9 & 0.8 & 0.8 & 1.2 & 0.6 & 0.9 & 0.4 & 0.3 & 0.7 & 0.3 \\
\hline Bike & 3.6 & 3.4 & 4.2 & 7.8 & 10.8 & 2.5 & 2.5 & 4 & 5.8 & 9.9 & 1.9 & 2.1 & 4.7 & 7.9 & 22.2 \\
\hline Sum Public & 27.1 & 40.9 & 52.5 & 57.3 & 69.2 & 11.7 & 17.4 & 31.9 & 38.3 & 54.5 & 0.9 & 1.7 & 1.7 & 8.6 & 9.9 \\
\hline Bus & 2.6 & 1.6 & 1.3 & 3.7 & 1.3 & 3.1 & 3.6 & 3 & 7.6 & 4.6 & 0.3 & 1.7 & 1.6 & 8.5 & 8.9 \\
\hline Train & 20.4 & 21.7 & 26.8 & 29.6 & 31.8 & 6.4 & 7.7 & 17 & 19.9 & 24.1 & 0.6 & 0 & 0.1 & 0.1 & 1 \\
\hline S-train & 1.9 & 2.6 & 10.2 & 5.2 & 14.3 & 2.2 & 6.1 & 11.9 & 10.8 & 25.9 & 0 & 0 & 0 & 0 & 0 \\
\hline Metro & 2.2 & 15 & 14.2 & 18.8 & 21.7 & 0 & 0 & 0 & 0 & 0 & 0 & 0 & 0 & 0 & 0 \\
\hline
\end{tabular}

The darker color represents better effect towards decarbonization. 
Table 7

Comparing the modal share potential across scenarios in 2050 aggregated based on income groups (percentage).

\begin{tabular}{|c|c|c|c|c|c|c|c|c|c|c|c|c|c|c|c|c|c|c|c|c|}
\hline & \multicolumn{5}{|c|}{ Very Low $\leq 200 \mathrm{kDKK} /$ year } & \multicolumn{5}{|c|}{$200<$ Low $\leq 500$} & \multicolumn{5}{|c|}{$500<$ Medium $\leq 800$} & \multicolumn{5}{|c|}{$800<$ High } \\
\hline & BAU & EPI & ISM & DPC & $\mathrm{COM}$ & BAU & EPI & ISM & DPC & $\mathrm{COM}$ & BAU & EPI & ISM & DPC & $\mathrm{COM}$ & BAU & EPI & ISM & DPC & $\mathrm{COM}$ \\
\hline Private & 83.3 & 75.8 & 65.1 & 45.9 & 22.8 & 86.5 & 81.5 & 72.2 & 65.5 & 51.5 & 86.2 & 81.9 & 73.2 & 71 & 60 & 80.5 & 74.4 & 62.7 & 63.5 & 51.2 \\
\hline $\begin{array}{l}\text { Sum Non- } \\
\text { motorized }\end{array}$ & 5 & 4.5 & 8.1 & 15.6 & 30.9 & 2.7 & 2.4 & 3.7 & 4.7 & 8.4 & 2.3 & 2.1 & 2.5 & 2.7 & 3.7 & 2.2 & 2 & 2.2 & 2.2 & 2.9 \\
\hline Walk & 1.5 & 1 & 1.1 & 1.3 & 0.6 & 0.8 & 0.4 & 0.5 & 1.1 & 0.5 & 0.2 & 0 & 0.1 & 0.3 & 0.1 & 0.1 & 0 & 0.1 & 0.1 & 0 \\
\hline Bike & 3.5 & 3.5 & 7 & 14.3 & 30.3 & 2 & 2 & 3.2 & 3.6 & 7.9 & 2.1 & 2.1 & 2.4 & 2.4 & 3.6 & 2 & 2 & 2.1 & 2.1 & 2.9 \\
\hline Sum Public & 11.6 & 19.7 & 26.8 & 38.5 & 46.3 & 10.8 & 16.1 & 24.1 & 29.8 & 40.2 & 11.5 & 15.9 & 24.3 & 26.3 & 36.3 & 17.3 & 23.6 & 35.1 & 34.3 & 45.9 \\
\hline Bus & 3.9 & 5.2 & 4.7 & 15.3 & 12 & 0.9 & 0.7 & 0.3 & 2.9 & 2.3 & 0.6 & 0.4 & 0.2 & 0.7 & 0.3 & 0.6 & 0.4 & 0.2 & 0.5 & 0.1 \\
\hline Train & 4.6 & 4.3 & 11.1 & 10.6 & 14.2 & 8.1 & 8.9 & 13.4 & 16.2 & 18.3 & 10 & 11.1 & 13.9 & 17.5 & 18.8 & 15.6 & 17.4 & 20.1 & 23.5 & 24.9 \\
\hline S-train & 2.5 & 5.6 & 6.6 & 7 & 14 & 1.1 & 2 & 6.2 & 5 & 12.6 & 0.4 & 0.8 & 6.9 & 3.6 & 11.5 & 0.2 & 0.3 & 9.3 & 3.1 & 12.8 \\
\hline Metro & 0.6 & 4.6 & 4.4 & 5.6 & 6.1 & 0.6 & 4.5 & 4.2 & 5.8 & 7 & 0.5 & 3.6 & 3.3 & 4.5 & 5.6 & 0.9 & 5.5 & 5.5 & 7.1 & 8.2 \\
\hline
\end{tabular}

The darker color represents better effect towards decarbonization.

with the network. Moreover, the methodology requires extensive survey data to define the characteristics of consumers, which are input to the model as agents' attributes and could be challenging to acquire in some countries. The modal perception of the agents, which drives their modal choice is represented in a simplified way with respect to the traditional utility functions (Train, 1986). Perhaps the model could be developed by calculating the probability of derived utility over all alternative choices such as logit function. The modal perceptions are represented in the model as tangible costs and value of travel time, which are calculated with data widely available (ticket price, fuel price, vehicle taxes, value of time, average speed of each mode, average congestion time, access/egress and waiting time). From an energy-environmental perspective, the model does not account energy consumption nor any kind of emission. From an economic perspective, the model does not track the investment costs of the transport technologies nor those of the transport infrastructure. However, these limitations do not affect the objective of this study.

Overall, the methodology adopted for this study allows for an analysis of how modal shift occurs as consequence of certain policies in a fast, reliable and scalable way, while the availability of the data required makes it replicable for any other geographical context. The energy-environmental-economic limitations identified can be addressed in future research by soft-linking the ABMoS-DK model with an E4 technology-rich energy optimization model, e.g. TIMES-DK (Balyk et al., 2019). The soft-link with TIMES-DK could open a new prospect to better represent the implications of human behaviour on the transport sector, evaluating the influence of modal shift on the future development of the energy system and the contribution of integrating behavioural aspects within the context of modal shift to the decarbonization of the energy system.

\subsection{Policy insights}

The analyses carried out within this study are meant to suggest to Danish policy makers which policy levers should be implemented to encourage a shift from private car to less carbon-intensive modes, such as non-motorized and public transport. This study has analyzed the modal shift resulting from a range of policy measures affecting the level of service of the modes. Public transport and non-motorized modes compete with car in different trip distances: the study found that metro, bicycle and walk are valid substitutions to car in short distance, while train, S-train and bus in long distance. The analyses discovered that for Denmark the highest shift away potential from car lies in urban areas, where more modal alternatives are available. Moreover, very low and low income groups are more receptive of the policies analyzed in this study and are most willing to shift away from car.

\section{Conclusions}

Agent-based modeling is capable of simulating the mode choice behaviour and perceptions of a large number of heterogeneous individuals with different characteristics. This study presents ABMoS-DK, a novel agent-based modeling approach that allows to evaluate the modal split for the inland passenger transportation sector in Denmark, which is able to capture a fine resolution of rational behavioural heterogeneity. The socio-economic characteristics of heterogeneous agents are taken from Danish national travel survey and the characteristics of modes are formulized to calculate tangible costs and value of travel time using LTM as a supporting model. The agents as rational decisionmakers determine the level of importance of cost versus time and choose the mode with highest utility to meet travel demand and the model determines the maximum shift potential from the viewpoint of travelers while interacting with the network. The model determines endogenously the modal shares from 2010 until 2050 by simulating level of service of modes and consumers' rational behaviour to understand current transport modal distributions, factors affecting the mode choice decisions, and opportunities for improving network performance through a number of policy scenarios.

The analysis of the trend of modal split of the alternative scenarios points out that introducing effective taxation schemes, parking pricing and toll collection; decreasing the public transit ticket price, park and ride facilities and charging infrastructure for electric bikes; increasing the frequency and expansion of public transit infrastructure are applicable measures for encouraging travelers to shift away from car use. The results of the scenario analysis suggest that implementing analyzed scenarios in Denmark east are more effective to reduce car use compared to west. Moreover, the major potential to shift away car use is for long distance trips while the analyzed policies are more effective in urban areas where more modal alternatives are available. Finally, very low and low income groups are more receptive of the policies analyzed in this study and are more willing to shift away from car. This result suggests that policy makers shall first target the most sensitive consumer groups.

We find that expanding the public transport infrastructure provide comparative advantages over other modes and reinforces the performance of public transit through decreasing travel time. Therefore, the travelers are encouraged to reduce car use by $7.1 \%$ in 2050 compared to the reference scenario and shift to public modes. Incentivizing sustainable modes decreases the tangible cost of public transit and electric bikes and encourage the travelers to use more sustainable modes of transport and reduce car use by $19.0 \%$ in 2050 compared to the reference scenario. The latter scenario is more successful than the former scenario due to the perception of travelers and their willingness to pay less money rather than spend less time. Changing the Danish vehicle 
registration tax increases the total purchase price of private cars and consequently disincentivize the use of private cars and according to our analysis, could reduce car use by $29.8 \%$ in 2050 compared to BAU. The higher reduction potential of car use by disincentivising private cars compared to other scenarios might be due to the fact that travelers prefer to avoid paying more cost rather than receiving subsidies or spending more time. The various strategies are "complementary" (May et al., 2006) when combined; meaning that their combined implementation shifts more demand from car than each policy alone. Under an ambitious policy package to move away from private cars, Denmark has the potential to nearly cut car use in half (49.3\%) by 2050 compared to BAU.

The proposed ABMoS framework could be used by policy makers to analyze the potential modal shift resulting from imposing policies in different geographical zones, targeting specific consumer groups (with similar characteristics concerning e.g., age, income, gender, education level, car ownership) and certain trip purposes in the long-term. By representing the heterogeneity of consumers' preferences and rational behaviour regarding mode choice simulation, policy makers could better understand the dynamics of the transportation system and target consumer groups with the highest shift potential. This paper contributes to producing a plan for achieving the 2050 decarbonization target within the Danish inland passenger transport sector by capturing rational behavioural realism of consumers.

\section{Acknowledgements}

This article and latest model development efforts are done within the Nordic Flagship Project SHIFT (Sustainable Horizons for Transport) project (grant number 77892), financed by Nordic Energy Research, Norway, and the COMETS (Co-Management of Energy and Transport Sector) project (grant number 4106-00033A) which has received funding from Innovation Fund Denmark, Denmark. The authors would like to acknowledge Professor Jeppe Rich and Søren Hasling Pedersen from Transport DTU for providing the data of LTM. We also acknowledge Hjalmar Christiansen from Transport DTU who has kindly made the data from the Danish National Travel Survey available and Mehran Ghainian Hasarouye who has helped with enhancing the performance and speed of Java code.

\section{Appendix A. Supplementary data}

Supplementary data to this article can be found online at https:// doi.org/10.1016/j.cstp.2019.07.010.

\section{References}

Adelt, F., Weyer, J., Hoffmann, S., Ihrig, A., 2018. simulation of the governance of complex systems (SimCo): basic concepts and experiments on urban transportation. J. Artif. Soc. Soc. Simul. 21 (2), 2. https://doi.org/10.18564/jasss.3654.

Ahanchian, M., Biona, J.B.M., 2017. Modeling rational, psychological and social behaviour towards diffusion of new technology using agent based simulation: The case of the public utility jeepney (PUJ) fleet in Metro Manila. J. Adapt. Behav. 25 (4), 165-183. https://doi.org/10.1177/1059712317716264.

Arbués, P., Baños, J.F., Mayor, M., Suárez, P., 2016. Determinants of ground transport modal choice in long-distance trips in Spain. Transport. Res. Part A: Policy Practice 84, 131-143. https://doi.org/10.1016/j.tra.2015.06.010.

Baindur, D., Viegas, J.M., 2011. An agent based model concept for assessing modal share in inter-regional freight transport markets. J. Transp. Geogr. 19 (6), 1093-1105. https://doi.org/10.1016/j.jtrangeo.2011.05.006.

Balyk, O., Andersen, S.K., Dockweiler, S., Gargiulo, M., Karlsson, K., Næraa, R., Petrović, S., Tattini, J., Termansen, L.B., Venturini, G., 2019. TIMES-DK: Technology-rich multi-sectoral optimisation model of the Danish energy system. Energy Strategy Rev. 23, 13-22. https://doi.org/10.1016/j.esr.2018.11.003.

Ben-Dor, G., Ben-Elia, E., Benenson, I., 2018. Assessing the impacts of dedicated bus lanes on urban traffic congestion and modal split with an agent-based model. Proc. Comput. Sci. 130, 824-829. https://doi.org/10.1016/j.procs.2018.04.071.

Boateng, M.K., Awuah-Offei, K., 2017. Agent-based modeling framework for modeling the effect of information diffusion on community acceptance of mining. Technol. Forecast. Soc. Chang. 117, 1-11. https://doi.org/10.1016/j.techfore.2017.01.019. Bonabeau, E., 2002. Agent-based modeling: Methods and techniques for simulating human systems. Proc. Natl. Acad. Sci. 99 (3), 7280-7287. https://doi.org/10.1073/ pnas.082080899.

Borshchev, A., Karpov, Y., Kharitonov, V., 2002. Distributed simulation of hybrid systems with AnyLogic and HLA. Future Gener. Comput. Syst. 18 (6), 829-839. https://doi. org/10.1016/S0167-739X(02)00055-9.

Barisa A., 2016. Modeling transportation policies to a low-carbon road transport in Latvia by 2030 . Doctoral thesis.

Borshchev A., Kolesov B., Senichenkov Y., 2000. Java engine for UML-based hybrid state machines, in: Proceedings of the Winter Simulation Conference. 10-13 December, Orlando, FL, USA. doi:10.1109/WSC.2000.899183.

Boyce D., 2005. Transportation systems in Encyclopedia of Life Support Systems, T. J. Kim, Ed. Oxford.

Brand, C., Tran, M., Anable, J., 2012. The UK transport carbon model: an integrated life cycle approach to explore low carbon futures. Energy Policy 41, 107-124. https:// doi.org/10.1016/j.enpol.2010.08.019.

Can, V.V., 2013. Estimation of travel mode choice for domestic tourists to Nha Trang using the multinomial probit model. Transport. Res. Part A: Policy Practice 49, 149-159. https://doi.org/10.1016/j.tra.2013.01.025.

Chen Z., Liu, X. C., 2018. Agent-Based Approach to Analyzing the Effects of Dynamic Ridesharing in a Multimodal Network. Transportation Research Board 97th Annual Meeting. Washington DC, United States. http://amonline.trb.org/.

Chikaraishi, M., Nakayama, S., 2016. Discrete choice models with q-product random utilities. Transport. Res. Part B: Method. 93 (Part A), 576-595. https://doi.org/10. 1016/j.trb.2016.08.013.

Chiodi, A., Gargiulo, M., Rogan, F., Deane, J.P., Lavigne, D., Rout, U.K., Ó Gallachóir, B., 2013. Modelling the impacts of challenging 2050 European climate mitigation targets on Ireland's energy system. Energy Policy 53, 169-189. https://doi.org/10.1016/j. enpol.2012.10.045.

Christiansen H., Skougaard B.Z., 2015. Documentation of the Danish National Travel Survey, Report 10, DTU Transport, Data and model center, ISSN 1601-9458.

Daly, H.E., Ramea, K., Chiodi, A., Yeh, S., Gargiulo, M., Ó Gallachóir, B., 2014. Incorporating travel behaviour and travel time into TIMES energy system models. Appl. Energy 135, 429-439. https://doi.org/10.1016/j.apenergy.2014.08.051.

Dia, H., 2002. An agent-based approach to modelling driver route choice behaviour under the influence of real-time information. Transport. Res. Part C: Emerg. Technol. 10 (5-6), 331-349. https://doi.org/10.1016/S0968-090X(02)00025-6.

Djavadian, S., Chow, J.Y.J., 2017. An agent-based day-to-day adjustment process for modeling 'Mobility as a Service' with a two-sided flexible transport market. Transport. Res. Part B: Method. 104, 36-57. https://doi.org/10.1016/j.trb.2017.06. 015.

E3MLab, 2014. ICCS at National Technical University of Athens. PRIMES-TREMOVE Transport Model, Detailed model description.

Eboli, L., Forciniti, C., Mazzulla, G., Calvo, F., 2016. Exploring the factors that impact on transit use through an ordered probit model: the case of metro of Madrid. Transp. Res. Proc. 18, 35-43. https://doi.org/10.1016/j.trpro.2016.12.005.

Eurostat. European cities - The EU-OECD functional urban area definition ,http://ec. europa.eu/eurostat/statistics-explained/index.php/European_cities_\%E2\%80\%93_ the EU-OECD functional urban area definition; 2017 [accessed 24.1.2017].

Fagnant, D.J., Kockelman, K.M., 2014. The travel and environmental implications of shared autonomous vehicles, using agent-based model scenarios. Transport. Res. Part C: Emerg. Technol. 40, 1-13. https://doi.org/10.1016/j.trc.2013.12.001.

FDM., 2017. Forenede Danske Motorejere, Billigere at køre efter rundstykker. http:// fdm.dk.

Føyn, T.H.Y., Karlsoon, K., Balyk, O., Grohnheit, P.E., 2011. A global renewable energy system: A modelling exercise in ETSAP/TIAM. Appl. Energy 88 (2), 526-534. https:// doi.org/10.1016/j.apenergy.2010.05.003.

GEA writing team, 2012. Global Energy Assessment: Toward a Sustainable Future Cambridge University Press, Cambridge.

Girod, B., van Vuuren, D.P., Deetman, S., 2012. Global travel within the $2{ }^{\circ} \mathrm{C}$ climate target. Energy Policy 45, 152-166. https://doi.org/10.1016/j.enpol.2012.02.008.

Hager, K., Rauh, J., Rid, W., 2015. Agent-based modeling of traffic behaviour in growing metropolitan areas. Transp. Res. Proc. 10, 306-315. https://doi.org/10.1016/j.trpro. 2015.09.080.

Hammadou, H., Papaix, C., 2015. Policy packages for modal shift and CO2 reduction in Lille, France. Transport. Res. Part D: Transport Environ. 38, 105-116. https://doi. org $/ 10.1016 / \mathrm{j} . \operatorname{trd} .2015 .04 .008$.

Hörl, S., Balac, M., Axhausen, K.W., 2018. A first look at bridging discrete choice modeling and agent-based microsimulation in MATSim. Proc. Comput. Sci. 130, 900-907. https://doi.org/10.1016/j.procs.2018.04.087.

International Energy Agency, 2009. Transport, Energy and CO2. IEA-Publications, Paris.

International Energy Agency, 2016. Nordic Energy Technology Perspectives 2016. Cities, flexibility and pathways to carbon-neutrality. http://www.nordicenergy.org/wpcontent/uploads/2016/04/Nordic-Energy-Technology-Perspectives-2016.pdf.

Jensen, S.S., Ketzel, M., Becker, T., Christensen, J., Brandt, J., Plejdrup, M., Winther, M., Nielsen, O.K., Hertel, O., Ellermann, T., 2017. High resolution multi-scale air quality modelling for all streets in Denmark. Transport. Res. Part D: Transport Environ. 52 (Part A), 322-339. https://doi.org/10.1016/j.trd.2017.02.019.

Kaddoura, I., Nagel, K., 2016. Agent-based congestion pricing and transport routing with heterogeneous values of travel time savings. Proc. Comput. Sci. 83, 908-913. https:// doi.org/10.1016/j.procs.2016.04.184.

Lu, X.S., Liu, T.L., Huang, H.J., 2015. Pricing and mode choice based on nested logit model with trip-chain costs. Transp. Policy 44, 76-88. https://doi.org/10.1016/j. tranpol.2015.06.014.

Macal, C.M., North, M.J., 2010. Tutorial on agent-based modelling and simulation. J. Simul. 4, 151-162. https://doi.org/10.1057/jos.2010.3.

Maciejewski, M., Nagel, K., 2011. Towards multi-agent simulation of the dynamic vehicle 
routing problem in MATSim. In: Wyrzykowski, R., Dongarra, J., Karczewski, K., Waśniewski, J. (Eds.), Parallel Processing and Applied Mathematics. PPAM 2011. Lecture Notes in Computer Science, vol. 7204. Springer, Berlin, Heidelberg.

Maggi, E., Vallino, E., 2016. Understanding urban mobility and the impact of public policies: The role of the agent-based models. Res. Transport. Econ. 55, 50-59. https://doi.org/10.1016/j.retrec.2016.04.010.

Mallig, N., Kagerbauer, M., Vortisch, P., 2013. mobiTopp - A modular agent-based travel demand modelling framework. Proc. Comput. Sci. 19, 854-859. https://doi.org/10. 1016/j.procs.2013.06.114.

Malokin, A., Circella, G., Mokhtarian, P.L., 2015. How Do activities conducted while commuting influence mode choice? Testing public transportation advantage and autonomous vehicle scenarios. In: 94th Annual Meeting of the Transportation Research Board.

Martinez, L.M., Correia, G.H.A., Viegas, J.M., 2014. An agent-based simulation model to assess the impacts of introducing a shared-taxi system: an application to Lisbon (Portugal). J. Adv. Transport. 49 (3), 475-495. https://doi.org/10.1002/atr.1283.

Martinez, L.M., Correia, G.H., Moura, F., Lopes, M.M., 2016. Insights into carsharing demand dynamics: outputs of an agent-based model application to Lisbon, Portugal. Int. J. Sustain. Transport. 436, 148-159. https://doi.org/10.1080/15568318.2016. 1226997.

Martinez, L.M., Viegas, J.M., 2017. Assessing the impacts of deploying a shared selfdriving urban mobility system: an agent-based model applied to the city of Lisbon, Portugal. Int. J. Transp. Sci. Technol. 6 (1), 13-27. https://doi.org/10.1016/j.ijtst. 2017.05.005.

Mathiesen, B.V., Kappel, J., 2013. Transport Policies Related to Climate Change Mitigation: The Case of Denmark. Department of Development and Planning, Aalborg University.

May, A.D., Kelly, C., Shepherd, S., 2006. The principles of integration in urban transport strategies. Transp. Policy 13 (4), 319-327. https://doi.org/10.1016/j.tranpol.2005. 12.005

McCollum, D., Yang, C., Yeh, S., Ogden, J., 2012. Deep greenhouse gas reduction scenarios for California-Strategic implications from the CA-TIMES energy-economic systems model. Energy Strategy Rev. 1 (1), 19-32. https://doi.org/10.1016/j.esr 2011.12.003.

McFadden D., 1978. Modeling the choice of residential location Transportation Research Record. 672, 72-77.

Mobycon, 2014. Electric Bicycle Report. http://supercykelstier.dk/wp-content/uploads/ 2016/03/El-cykelrapport h\%C3\%B8j_opl\%C3\%B8sning.compressed-2.pdf.

Novosel, T., Perković, L., Ban, M., Keko, H., Pukšec, T., Krajačić, G., Duić, N., 2015. Agent based modelling and energy planning - Utilization of MATSim for transport energy demand modelling. Energy 92, 466-475. https://doi.org/10.1016/j.energy.2015.05. 091.

Pye, S., Daly, H., 2015. Modelling sustainable urban travel in a whole systems energy model. Appl. Energy 159, 97-107. https://doi.org/10.1016/j.apenergy.2015.08.127.

Qu, Y., Zhou, X., 2017. Large-scale dynamic transportation network simulation: A spacetime-event parallel computing approach. Transport. Res. Part C: Emerg. Technol. 75, 1-16. https://doi.org/10.1016/j.trc.2016.12.003.

Rich, J., 2015. The weekday demand model in LTM - model for generation. Destin. Mode Choice 1-159.

Rich, J., Hansen, C.H., 2015. The Danish National Passenger Model. Working Paper Dec 2015. Eur. J. Transport Infrastruct. Res. https://doi.org/10.13140/RG.2.1.3661. 7040.

Schäfer A., 2012. Introducing Behavioural Change in Transportation into Energy/ Economy/Environment Models. Draft Report for Green Development Knowledge Assessment of the World Bank.

Schäfer, A., Victor, D.G., 2000. The future mobility of the world population. Transport. Res. Part A: Policy Practice 34 (3), 171-205. https://doi.org/10.1016/S09658564(98)00071-8.

Scheltes, A., Correia, G.H.D.A., 2017. Exploring the use of automated vehicles as last mile connection of train trips through an agent-based simulation model. Int. J. Transp. Sci. Technol. 6 (1), 28-41. https://doi.org/10.1016/j.ijtst.2017.05.004.

Shafiei, E., Thorkelsson, H., Ásgeirsson, E.I., Davidsdottir, B., Raberto, M., Stefansson, H., 2012. An agent-based modeling approach to predict the evolution of market share of electric vehicles: A case study from Iceland. Technol. Forecast. Soc. Chang. 79 (9),
1638-1653. https://doi.org/10.1016/j.techfore.2012.05.011.

Shirzadi-Babakan, A., Alimohammadi, A., Taleai, M., 2015. An agent-based evaluation of impacts of transport developments on the modal shift in Tehran. Iran. J Dev Effect. 7 230-251. https://doi.org/10.1080/19439342.2014.994656.

Shirzadi-Babakan, A., Taleai, M., 2015. Impacts of transport development on residence choice of renter households: An agent-based evaluation. Habitat Int. 49, 275-285. https://doi.org/10.1016/j.habitatint.2015.05.033.

Shukla, N., Munoz, A., Ma, J., Huynh, N., 2013. Hybrid agent based simulation with adaptive learning of travel mode choices for university commuters. Paper presented at the Workshop on Model-driven Approaches for Simulation Engineering, Symposium on Theory of Modeling and Simulation, SCS SpringSim Conference, San Diego, CA, USA (2013, April).

Sims R., Schaeffer R., Creutzig F., Cruz-Núñez X., D’Agosto M., Dimitriu D., Figueroa Meza M.J., Fulton L., Kobayashi S., Lah O., McKinnon A., Newman P., Ouyang M., Schauer J.J., Sperling D., Tiwari G., 2014. Transport. In: Climate Change 2014: Mitigation of Climate Change. Contribution of Working Group III to the Fifth Assessment Report of the Intergovernmental Panel on Climate Change [Edenhofer, O., R. Pichs-Madruga, Y. Sokona, E. Farahani, S. Kadner, K. Seyboth, A. Adler, I. Baum, S. Brunner, P. Eickemeier, B. Kriemann, J. Savolainen, S. Schlömer, C. von Stechow, T. Zwickel and J.C. Minx (eds.)]. Cambridge University Press, Cambridge, United Kingdom and New York, NY, USA.

Sopha, B.M., Klockner, C.A., Hertwich, E.G., 2011. Exploring policy options for a transition to sustainable heating system diffusion using an agent-based simulation. Energy Policy 39, 2722-2729. https://doi.org/10.1016/j.enpol.2011.02.041.

Statistics Denmark, n.d. Population projections - Table FRDK117; 2017. http://www. statistikbanken.dk/1920 [accessed 10.4.2017].

Tattini, J., Gargiulo, M., Karlsson, K., 2018a. Reaching carbon neutral transport sector in Denmark - Evidence from the incorporation of modal shift into the TIMES energy system modeling framework. Energy Policy 113, 571-583. https://doi.org/10.1016/ j.enpol.2017.11.013.

Tattini, J., Ramea, K., Gargiulo, M., Yang, C., Mulholland, E., Yeh, S., Karlsson, K., 2018b. Improving the representation of modal choice into bottom-up optimization energy system models - The MoCho-TIMES model. Appl. Energy 212, 265-282. https://doi. org $/ 10.1016 /$ j.apenergy.2017.12.050.

The official website of Denmark, 2017. http://denmark.dk/en/green-living/strategiesand-policies/independent-from-fossil-fuels-by-2050.

Thrane, C., 2015. Examining tourists' long-distance transportation mode choices using a Multinomial Logit regression model. Tour. Manage. Perspect. 15, 115-121. https:// doi.org/10.1016/j.tmp.2014.10.004

Train, K., 1986. In: Qualitative Choice Analysis: Theory, Econometrics, and an Application to Automobile Demand. The MIT Press, Cambridge.

TU Survey 2018. https://www.cta.man.dtu.dk/english/tvu/latest_dataset. [accessed 17. 12.2018].

Venturini, G., Tattini, J., Mulholland, E., Ó Gallachóir, B.P., 2018. Improvements in the representation of behavior in integrated energy and transport models. Int. J. Sustainable Transport. https://doi.org/10.1080/15568318.2018.1466220.

Waisman, H., Guivarch, C., Lecocq, F., 2013. The transportation sector and low carbon growth pathways: modeling urban, infrastructure and spatial determinants of mobility. Climate Policy 13, 106-129. https://doi.org/10.1080/14693062.2012. 735916

Winther, M., 2015. Danish Emission Inventories for Road Transport and Other Mobile Sources. Aarhus University, Danish Centre for Environment and Energy.

Yang, C., Yeh, S., Zakerinia, S., Ramea, K., McCollum, D., 2015. Achieving California's $80 \%$ greenhouse gas reduction target in 2050: Technology, policy and scenario analysis using CA-TIMES energy economic systems model. Energy Policy 77, 118-130. https://doi.org/10.1016/j.enpol.2014.12.006.

Ziemke, D., Metzler, S., Nagel, K., 2018. Bicycle traffic and its interaction with motorized traffic in an agent-based transport simulation framework. Future Gen. Comput. Syst. https://doi.org/10.1016/j.future.2018.11.005.

Zou, M., Meng, L., Xi, L., Xiong, C., Mao, C., Cheng, W., Ke, Zhang, Jiaying, Y., 2016. An agent-based choice model for travel mode and departure time and its case study in Beijing. Transport. Res. Part C: Emerg. Technol. 64, 133-147. https://doi.org/10. 1016/j.trc.2015.06.006 